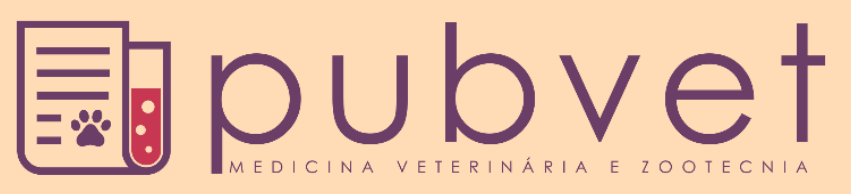

https://doi.org/10.31533/pubvet.v15n06a831.1-12

\title{
Reconhecimento e avaliação da dor em bovinos: Revisão
}

\author{
Marllos Henrique Vieira Nunes $^{1 *}$, Acácio Duarte Pacheco ${ }^{2} \bullet$ (D) Juliana Tessália Wagatsuma $^{3} \bullet$ \\ ${ }^{I}$ Discente, Programa de Pós Graduação em Sanidade e Produção Animal Sustentável na Amazônia Ocidental, Universidade Federal do Acre, Rio Branco-Ac, Brasil. \\ ${ }^{2}$ Professor da Universidade Federal do Acre, Departamento de Ciências da Natureza, Rio Branco-Ac, Brasil. \\ ${ }^{3}$ Médica Veterinária Anestesiologista, Universidade Federal do Acre, Rio Branco-Ac, Brasil. \\ *Autor para correspondência, E-mail: marllosvet11@gmail.com
}

Resumo. A dor, além de ser uma experiência desagradável, é um mecanismo importante de proteção do organismo e manutenção da vida, e o entendimento da sua fisiologia, suas principais causas, seu reconhecimento e avaliação em bovinos é necessário para o direcionamento do tratamento ideal, que promova o bem-estar animal minimizando os prejuízos na cadeia produtiva. A avaliação da dor em bovinos representa um desafio e o seu tratamento é considerado muitas vezes inadequado, devido à falta de conhecimento sobre os comportamentos relacionados a dor e a crença que pela sua rusticidade e resistência sintam menos dor que os pequenos animais, ou até mesmo não sentem dor. Os estudos a respeito da avaliação da com base no comportamento tem recebido atenção crescente, e geralmente comportamentos de dor em bovinos descritos na literatura são aqueles frequentemente associados a doenças como mastites, metrites, pododermatites, artrite séptica, peritonite, miíases externas, ou práticas de manejo como castrações, marcação com ferro quente e descornas. Algumas ferramentas, como escalas de avaliação da dor, estão sendo produzidas e utilizadas para a avaliação da dor em bovinos, e é notória a percepção da sociedade sobre a importância do reconhecimento e tratamento da dor não só em bovinos, mas em todos os animais de produção, objetivando sobretudo o bem-estar animal.

Palavras-chave: Bem-estar, comportamento, produção, ruminantes

\section{Recognition and assessment of pain in cattle: Review}

Abstract: Pain, in addition to being an unpleasant experience, is an important mechanism for protecting the organism and maintaining life, and an understanding of its physiology, its main causes, its recognition and evaluation in cattle is necessary to guide the ideal treatment, which promotes animal welfare by minimizing losses in the production chain. The assessment of pain in cattle represents a challenge and its treatment is often considered inadequate, due to the lack of knowledge about behaviors related to pain and the belief that due to its rusticity and resistance they feel less pain than small animals, or even don't have pain. Studies regarding behavioral assessment have received increasing attention, and generally pain behaviors in cattle described in the literature are those frequently associated with diseases such as mastitis, metritis, pododermatitis, septic arthritis, peritonitis, external myiasis, or handling such as castration, branding and dehorning. Some tools, such as pain assessment scales, are being produced and used to assess pain in cattle, and society's perception of the importance of recognizing and treating pain is not only notable in cattle, but in all animals. production, aiming mainly at animal welfare.

Keyword: well-being, behavior, production, ruminants 


\title{
Reconocimiento y evaluación del dolor en el ganado: Revisión
}

\begin{abstract}
Resumen. El dolor, además de ser una experiencia desagradable, es un mecanismo importante para proteger al organismo y mantener la vida, y es necesario conocer su fisiología, sus principales causas, su reconocimiento y evaluación en el ganado para orientar el tratamiento ideal, que promueva el bienestar animal minimizando las pérdidas en la cadena de producción. La valoración del dolor en los bovinos representa un reto y su tratamiento muchas veces se considera inadecuado, por el desconocimiento de las conductas relacionadas con el dolor y la creencia de que por su rusticidad y resistencia sienten menos dolor que los animales pequeños, o incluso no sienten dolor. Los estudios relacionados con la evaluación del comportamiento han recibido una atención cada vez mayor y, en general, los comportamientos dolorosos en el ganado que se describen en la literatura son los que se asocian frecuentemente con enfermedades como mastitis, metritis, pododermatitis, artritis séptica, peritonitis, miasis externa o manejo como castración, marcado y descornado. Algunas herramientas, como las escalas de evaluación del dolor, se están produciendo y utilizando para evaluar el dolor en el ganado, y la percepción de la sociedad sobre la importancia de reconocer y tratar el dolor no solo es notable en el ganado, sino en todos los animales de producción, dirigida principalmente al bienestar animal.
\end{abstract}

Palabra clave: Bienestar, comportamiento, producción, rumiantes

\section{Introdução}

A dor, juntamente com a função cardiorrespiratória e a função térmica, é considerada o quinto sinal vital, relacionada a uma experiência emocional ou sensorial desagradável, associada ou não uma injúria tecidual potencial que produz respostas comportamentais, neuroendócrinas, autonômicas e imunológicas. Em animais de produção pode gerar resultados negativos sob o desenvolvimento, consumo de alimentos, produtividade e bem-estar animal (Antunes et al., 2008).

A avaliação da dor em bovinos representa um desafio e, atrelado a isso, o tratamento da dor nesses animais é considerado muitas vezes inadequado, devido à falta de conhecimento sobre os comportamentos relacionados a dor e a crença que, pela sua rusticidade e resistência, sintam menos dor que os pequenos animais (Lorena et al., 2013; Mathews et al., 2015). Em animais jovens, o tratamento em muitos casos também é considerado insatisfatório, justificado pela falsa ideia de que possuem maior limiar de dor que os adultos ou que são submetidos a procedimentos cirúrgicos menos traumáticos (Fajt et al., 2011). Comumente, muitos procedimentos cirúrgicos são realizados sem o uso de anestésicos ou analgésicos em bovinos. Estudos realizados com médicos veterinários americanos revelaram que $40 \%$ não utiliza algum analgésico após a descorna em bezerros de corte, com menos de seis meses de idade, e em castrações de bezerros de qualquer idade (Fajt et al., 2011). Lorena et al. (2013) afirmam que 84\% dos veterinários questionados em sua pesquisa acreditam que seu conhecimento a respeito da analgesia de bovinos é inadequado, e $20 \%$ destes não usam anti-inflamatórios, e quando relatado o uso de analgésicos no pré-operatório e pós-operatório de cirurgias abdominais em bovinos, apenas 59\% e 75\%, respectivamente, dos animais recebem alguma dose nesses períodos.

Nos bovinos a avaliação da dor realizada por mensuração de parâmetros fisiológicos não é considerado o melhor método para determinar presença de dor, uma vez que esses valores podem ser considerados inespecíficos e influenciados pelo estresse, ao qual são sensíveis, além desses parâmetros serem difíceis de aferir nas fazendas (Gleerup et al., 2015).

Nesse contexto, a avaliação da dor em bovinos com base no comportamento tem recebido atenção crescente, e geralmente comportamentos de dor em bovinos descritos na literatura são aqueles frequentemente associados às doenças como mastites, metrites, pododermatites, artrite séptica, peritonite, miíases externas, ou práticas de manejo como castrações e descornas (Davidson et al., 2015; Leslie \& Petersson-Wolfe, 2012; Moggy et al., 2017; Shearer et al., 2013; Stojkov et al., 2015). Esses comportamentos incluem mudanças na postura, como arqueamento do dorso, abaixamento da cabeça, claudicação severa, vocalização e modificação do comportamento social e alimentar (Chapinal et al., 2010; Millman, 2013; Moggy et al., 2017). Recentemente, pesquisas utilizando os mais diversos modelos animais, como pequenos ruminantes (McLennan et al., 2016), equinos (Dalla Costa et al., 
2014), coelhos (Keating et al., 2012), gatos (Evangelista et al., 2019) e camundongos (Langford et al., 2010), revelaram que mudanças de comportamento sutis, como alterações na expressão facial, são excelentes ferramentas para avaliação da dor em bovinos (Amaral et al., 2017; Gleerup et al., 2015; Müller et al., 2019).

Levando em consideração que a dor é um mecanismo importante de proteção do organismo e manutenção da vida, o entendimento da sua fisiologia bem como suas principais causas, seu reconhecimento e avaliação em bovinos é necessário para a tomada de decisão quanto ao melhor tratamento a ser instituído, objetivando o bem-estar animal e redução dos prejuízos na cadeia produtiva. Desta forma, essa revisão tem por objetivo elencar os principais processos desencadeadores de dor em bovinos e as ferramentas necessárias para a avaliação e seu correto manejo nessa espécie.

\section{Situações causadoras de dor em bovinos}

Diversos estudos envolvendo avaliação de dor em bovinos revelam que, além as de doenças como mastites, metrites, pododermatites, os bovinos também podem apresentar dor aguda em decorrência de estímulos nocivos associados a procedimentos zootécnicos de manejo e produção como castrações, descorna ou marcação a ferro quente (Fajt et al., 2011; González et al., 2010; Kaler et al., 2010; Laven et al., 2012; Lorena et al., 2013; Moggy et al., 2017; Roches et al., 2017; Stojkov et al., 2015). A aplicação de brincos, outra prática comum de manejo, quando não executada corretamente com o brinco introduzido no centro da orelha, pode gerar inflamação e até rasgar a orelha dos animais, o que causa dor e desconforto (Anzuino et al., 2010).

Distocias e intervenções obstétricas como cesarianas, também costumam ser relatadas como fatores geradores de dor em bovinos, principalmente quando não assistidas por profissionais capacitados e sem o devido manejo analgésico empregado (Moggy et al., 2017).

Amplamente utilizada como prática de manejo, principalmente em bezerros de corte em todo o mundo, a castração impõe danos físicos, químicos e hormonais aos testículos geralmente executada por métodos físicos, como a remoção cirúrgica ou pelo uso do burdizzo e aplicação de anéis elásticos colocados na região proximal do cordão espermático (Coetzee, 2013). Embora os benefícios da castração sejam consideravelmente aceitos na grande maioria dos países, como redução do comportamento de monta, agressividade, melhor qualidade da carne como maciez e marmoreio, todos os métodos de castração são capazes de produzir mudanças fisiológicas, neuroendócrinas e comportamentais que levam a dor e angústia (González et al., 2010). Grande parte das castrações são realizadas sem o devido uso de analgésicos, anti-inflamatórios não-esteroidais ou anestésicos locais, haja visto que por diversas vezes essa prática não é realizada por médicos veterinários, e mesmo quando são, pouca atenção é dada à analgesia pós-operatória (Antunes et al., 2008).

Uma pesquisa realizada com 109 produtores de vacas e bezerros no oeste canadense revelou que 95\% realizavam castração em bezerros com menos de 3 meses de idade, e que 90\% não utilizava alguma estratégia de mitigação de dor, como uso de anestésicos locais ou anti-inflamatórios (Moggy et al., 2017).

No Reino Unido, um estudo realizado via questionário coma 242 pecuaristas a respeito do uso de medicamentos analgésicos e atribuição de escores de intensidade de dor em bezerros submetidos a castração cirúrgica e descorna em bovinos adultos apontou má avaliação quanto ao valor atribuído a dor nos animais e que no máximo 30\% dos entrevistados usam anti-inflamatórios não esteroidais após os procedimentos (Remnant et al., 2017).

A descorna é uma prática comum realizada em bovinos de corte e de leite, implementada por diversos motivos, dentre eles, segurança no manejo, menor risco de acidentes entre os animais e menor exigência de espaço no comedouro (Stock et al., 2013). Entretanto, os métodos de descorna utilizados em ruminantes, cirúrgico, cauterização química e térmica, causam uma resposta de estresse agudo considerável, com aumento da concentração plasmática de cortisol, principalmente quando anestésicos locais, analgésicos e sedativos não são utilizados no procedimento (Stock et al., 2013). Estudos realizados em regiões do centro-norte e nordeste dos Estados Unidos com produtores de laticínios estaduais mostraram que apenas $12,4 \%$ destes usam alguma técnica de bloqueio anestésico local e 1,8\% usam analgésicos por via sistêmica no momento da descorna (Fulwider et al., 2008). Trabalhos 
comparando a descorna com a castração revelaram que a descorna resulta em mais respostas agudas ao estresse, de acordo com os resultados obtidos de concentração de cortisol, sob as mesmas condições experimentais. Nesses animais foi observado uma redução no ganho de peso diário nos bovinos que foram submetidos a descorna 2 a 3 semanas após a castração (Ballou et al., 2013; Mosher et al., 2013; Sutherland et al., 2013).

Durante o parto, algumas situações como distocias ou cesarianas podem desencadear experiências dolorosas nas vacas e nos fetos, principalmente quando mal gerenciadas com relação a analgesia. A distocia refere-se a um parto difícil, que necessita de assistência por diversos motivos, dentre eles a apresentação anterior do bezerro, seu grande porte e peso, e que resulta em parto prolongado e doloroso (Laven et al., 2012; Moggy et al., 2017).

Trabalhos relatam que ainda existem algumas dificuldades quanto a administração de analgésicos no período pós-parto de vacas leiteiras, sendo estas relacionadas a falhas na avaliação do grau de dor dos animais e a resistência quanto a utilização de substâncias farmacológicas que podem ser eliminadas pelo leite (McLennan et al., 2016). Animais que recebem analgesia pós-parto tendem a se alimentar mais rápido, bem como retomar suas atividades mais cedo, quando comparado a os que não recebem esse tratamento, o que destaca a importância de considerarmos o efeito da analgesia no pós-parto sobre o bem-estar animal e consequentemente sobre a produção (Newby et al., 2013; Stilwell et al., 2014).

A mastite, inflamação das glândulas mamárias, constitui-se como um dos principais problemas que afetam o bem-estar de vacas leiteiras, geralmente associadas a agentes infecciosos e erros de manejo na ordenha, comprometendo a qualidade do leite, causando dor intensa no úbere devido ao aumento da pressão intra-mamária pela inflamação. Dados apontam que até $23 \%$ do rebanho de vacas leiteiras pode ser acometido e que a doença pode evoluir de forma aguda podendo até levar o animal ao óbito (Leslie \& Petersson-Wolfe, 2012). Devido à inflamação e a dor, a mastite é capaz de induzir mudanças comportamentais e resposta ao estresse em vacas leiteiras após inoculação experimental por E. coli nas fases pré-clinica e aguda da doença, sendo estas mudanças consideradas efeitos negativos sobre o bemestar animal e que podem ser úteis para a detecção precoce de mastite e consequente tomada de decisão sobre o início do tratamento para alívio da dor (Roches et al., 2017).

A metrite é uma doença comum em vacas pós-parto associada a presença de dor visceral significativa e que possui taxa de incidência entre 10 e $30 \%$ em rebanhos bovinos, principalmente em fêmeas multíparas, gerando alterações comportamentais, redução no consumo de alimentos, ganho de peso e produção de leite (Giuliodori et al., 2013; Wittrock et al., 2011). Durante a fase clínica da doença o útero encontra-se aumentado de tamanho e é possível identificar a presença de secreção purulenta uterina e na vagina em até 21 dias após o parto, entretanto não há sinais de doença sistêmica, como febre ou sinais de toxemia (Opsomer, 2015). Um estudo conduzido por Stojkov et al. (2015) foi pioneiro ao avaliar a dor visceral como fator responsável pelas alterações comportamentais e fisiológicas de vacas leiteiras com metrite através de palpação retal com e sem palpação uterina, no pós-parto em período de tempo estabelecido, revelando que vacas com metrite apresentam maior sensibilidade uterina após palpação uterina via com palpação retal.

Doenças do casco, como as pododermatites, levam a claudicação grave e são frequentes em sistemas de criação de bovinos e pequenos ruminantes, sendo um forte indicativo de dor e podem atingir cerca de 21 a 37\% dos bovinos, chegando até 55\%, quando nos referimos aos animais destinados à produção de leite (Kaler et al., 2010; Randall et al., 2016; Shearer et al., 2013). Dados estimados apontam que em torno de $80 \%$ das vacas de leiteiras apresentam alguma afecção nos membros e, cerca de $25 \%$ destas, apresentam claudicação clínica em níveis variados (Bruijnis et al., 2012). A claudicação gera grandes impactos na indústria pecuária e perdas econômicas consideráveis, além de ser um fator que vai de encontro aos princípios de bem-estar animal na cadeia produtiva, pois está intimamente relacionada a dor e angústia nos animais, principalmente as de origem infeciosa (McLennan, 2018; Shearer et al., 2013). Prejuízos sobre a fertilidade de rebanhos de caprinos e bovinos, que contribuem para a toxemia da prenhez, doenças neonatais e incrementos na necessidade de abate precoce dos animais, também são consequências da claudicação, o que gera a necessidade de uma avaliação minuciosa, tratamento e correto manejo quanto a dor, que em muitos casos é proporcional à gravidade das afecções do casco ou membros acometidos (Battini et al., 2014). Shearer et al. (2013) afirmam que a claudicação em bovinos 
pode gerar alodinia e hiperalgesia, comum em casos de dermatite digital, em virtude de uma redução significativa nos limiares nociceptivos que pode persistir por até 28 dias após o início dos sinais, e quando não corretamente tratados, podem levar à ocorrência de dor crônica. Outros procedimentos realizados em bovinos são relatados na literatura como sendo potencialmente capazes de desencadear dor aguda nos animais, comprometendo seu bem-estar quando realizados sem o uso de estratégias de analgesia durante e após a execução dos mesmos, como correção de hérnias umbilicais, amputação de dígitos, fraturas distais de membros, úlceras de córnea e uveítes (Remnant et al., 2017).

\section{Alterações fisiológicas e comportamentais}

Para o reconhecimento e consequente tratamento da dor, são necessárias ferramentas úteis para a sua avaliação. Coetzee (2013) em seus estudos avaliou as mudanças fisiológicas, neuroendócrinas, como a liberação da substância P, e comportamentais induzidas pela dor. A dor é capaz de induzir uma série de respostas reflexas a partir do sistema nervoso central, que é o principal alvo de informação nociceptiva e que fornece subsídios para que o organismo possa reagir contra estímulos. Dentre essas respostas estão o aumento do tônus simpático que leva a uma vasoconstrição, aumento do débito cardíaco pelo aumento da frequência cardíaca e pressão arterial sistêmica, aumento do consumo de oxigênio pelo miocárdio, diminuição do tônus gastrointestinal e urinário e aumento do tônus do músculo esquelético (Cartopassi \& Junior, 2012).

É possível observar, como resposta endócrina um aumento da secreção de cortisol, do hormônio antidiurético $(\mathrm{ADH})$ e das catecolaminas circulantes, e uma diminuição da secreção de insulina e testosterona. Estas alterações levam a um estado catabólico caracterizado por hiperglicemia, retenção renal de água e sódio, com aumento da excreção de potássio e diminuição da taxa de filtração glomerular (Roches et al., 2017). No SNC, a dor pode resultar em hipoventilação, que culminará na ocorrência de taquipneia, interferindo diretamente na relação ventilação/perfusão. Além disso, a estimulação simpática gerada pode contribuir para um aumento da viscosidade sanguínea, do tempo de coagulação, agregação plaquetária e fibrinólise (Grimm et al., 2015). Todas essas respostas desencadeadas pela dor no organismo, nada mais são que uma condição adaptativa desenvolvida para otimizar a sobrevivência no período imediatamente após um estímulo doloroso. Entretanto, a persistência destas respostas pode tornar-se deletéria para o indivíduo, uma vez que essa resposta neuroendócrina à dor pós traumática ou pós-cirúrgica pode desencadear estado de choque no organismo (Coetzee, 2013).

O cortisol tem sido utilizado amplamente como uma medida de angústia e que pode indicar a intensidade e duração do estresse em animais de produção, estando o seu aumento relacionado com episódios de dor, entretanto, sua expressão também pode variar de acordo com o estímulo empregado, de acordo com o animal e o procedimento ao qual foi submetido (Coetzee, 2013).

Em bovinos submetidos à castração e descorna com uso de anestésicos locais ou analgésicos, comparados com animais submetidos aos mesmos procedimentos sem analgesia, o aumento do cortisol plasmático é atenuado (Stock et al., 2013). No entanto, estudos realizados em caprinos que passaram por descorna por cauterização e que receberam anestésico local para bloqueio do nervo cornual, não observaram redução da concentração de cortisol nos animais (Alvarez et al., 2015). Coetzee (2013) afirma que vários fatores podem levar a aumento do nível de cortisol nos bovinos, dentre eles o tipo de procedimento ao qual serão submetidos, o grau de invasão, o limiar de dor de cada animal, o método de castração empregado e até mesmo a pessoa que executa o procedimento, o que demonstra que nem sempre as concentrações de cortisol plasmático refletem com precisão a extensão da resposta da dor nos bovinos.

Dentre as alterações fisiológicas geradas pela dor, em bovinos, o aumento da frequência cardíaca, da pressão arterial e a redução da temperatura retal, é relatado (Stewart et al., 2010). Em um trabalho realizado com bezerros que passaram por descorna, foi possível observar um aumento na frequência cardíaca dos animais durantes os primeiros 30 segundos do procedimento (Jimenez et al., 2019). Em estudos que avaliaram o grau de claudicação de bovinos com sinovite, foi possível observar que aqueles animais que apresentavam grau de claudicação 2 e 3, possuíam frequência cardíaca maior que os bovinos com claudicação grau 1 (Kotschwar et al., 2009). 
Entretanto, na literatura existe uma variedade de trabalhos que evidenciam padrões de respostas diferentes com relação aos parâmetros fisiológicos quando avaliados em bovinos submetidos a algum estímulo doloroso, o que mostra que estes indicadores não têm aplicação prática em todas as situações clínicas, haja visto que muitas vezes a própria contenção do animal, diante do temperamento indócil, causa alterações cardiovasculares e endócrinas, não necessariamente relacionadas a dor (Galatos, 2011).

Trabalhos que utilizam pedômetros e acelerômetros, também são ferramentas úteis e que não requerem uma abordagem direta aos animais, pois viabilizam uma avaliação indireta da dor e estresse, tanto em situações experimentais como clínicas através da monitorização remota do comportamento frente aos estímulos gerados (Currah et al., 2009; White et al., 2008). O pedômetro é um equipamento que mede o número de passos, a postura, e relaciona essas medidas com o tempo e a frequência de decúbitos realizados pelos animais, sendo útil até mesmo na detecção de períodos reprodutivos, como o início do estro em vacas, mediante as alterações comportamentais expressas nesse período (Theurer et al., 2013). Em estudos com o uso de pedômetros mostraram que bezerros castrados sem anestésico local e anti-inflamatórios apresentaram menor número de passos após 24 horas do procedimento, em relação aos que receberam esses fármacos para analgesia (Currah et al., 2009). Também foi observado que garrotes se deitaram com maior frequência entre 1 e 4 horas após procedimento de orquiectomia, sedados com xilazina e bloqueio local com lidocaína (Oliveira et al., 2014).

Os acelerômetros mensuram de forma contínua o tempo gasto em pé e/ou em decúbito e o tempo de locomoção dos animais, através do processamento de valores da força gravitacional nos eixos horizontal, vertical e diagonal (Theurer et al., 2013). Bovinos que foram castrados, passaram menos tempo comendo e caminhando e apresentaram mais alterações em sua postura na posição quadrupedal e marcha, ou se deitaram com mais frequência com a cabeça próxima ao solo (Oliveira et al., 2014).

Outros estudos realizados em bezerros, mostraram que após castração e descorna, os animais passaram mais tempo deitados, por um período de até cinco dias após a cirurgia (Pauly et al., 2012). Já em outros estudos com bovinos adultos, através de mensurações com uso de acelerômetro, foi observado que após castração, esses animais passavam mais tempo em pé (González et al., 2010; White et al., 2008).

Outra ferramenta descrita na literatura utilizada para avaliação de dor em bovinos é o eletroencefalograma. Experimentos realizados em bovinos que foram submetidos a castração, revelaram um aumento significativo da atividade cerebral registrada pelo eletroencefalograma, devido ao estímulo de substâncias nocivas associadas a dor liberadas no momento do procedimento cirúrgico, em comparação ao período pré-cirúrgico (Bergamasco et al., 2009).

Em sua maioria, os métodos de avaliação da dor acima descritos, apresentam limitações, seja pelo fato de serem invasivos ou por exigirem algum equipamento específico para sua utilização, o que os restringe de serem implementados em situações clínicas rotineiras. Nesse contexto, a principal ferramenta para avaliação da dor em animais é o comportamento espécie-específico (Flecknell, 2008). Alterações comportamentais são mais fáceis de avaliar e, portanto, mais aplicáveis em situações clínicas. Entretanto, é necessário considerar que as respostas comportamentais à dor apresentam uma grande variação individual e não são constantes (Flecknell, 2008).

As alterações de comportamento mais comuns relacionadas à dor em bovinos são as mudanças na interação com o meio ambiente e com os outros animais, alterações na marcha, posição da cabeça, postura em posição quadrupedal e em decúbito, a atenção ao provável local da dor, lambeduras ou mordidas na área da ferida. Algumas mudanças na expressão facial podem ser percebidas, como os olhos cerrados. O apetite pode estar reduzido, assim como ruminação pode estar ausente ou diminuída e os animais tendem a perder peso (Futro et al., 2015; Guesgen et al., 2011; Millman, 2013; Oliveira et al., 2014; Stewart et al., 2010).

Após passarem por cirurgias de castração, é comum observar bovinos imóveis, com os membros pélvicos estendidos, ou afastados. Ao andarem possuem menor amplitude dos passos, e quando em pé, batem com o pé no chão e flexionam os membros pélvicos, movimentando de forma brusca a cauda, como se fosse chicotear, além de olharem para o local da lesão que possivelmente esteja gerando desconforto. $\mathrm{O}$ apetite e a ruminação também estão diminuídos. Tais comportamentos tendem a desaparecer ou serem atenuados quando analgésicos são administrados nesses animais (Millman, 2013). 
O método utilizado na orquiectomia influencia a manifestação de dor em bovinos. Por exemplo, animais castrados com burdizo costumam ficar mais ativos do que aqueles castrados cirurgicamente. Entretanto, a dor é pior nas primeiras três horas após castração, independentemente do método empregado (Coetzee, $\underline{2013}$ ).

Quando submetidos a descorna, os sinais etológicos mais característicos após o procedimento incluem o balançar da cabeça, movimentos de orelha, esfregar a cabeça contra superfícies, deitar-se e levantar-se com frequência, ficar deitado e imóvel e vocalizar. Tais comportamentos se manifestam em até 44 a 72 horas após a descorna (Stock et al., 2013).

Assim como os ovinos, nos bovinos a presença e o grau de claudicação podem ter forte correlação com a intensidade da dor e a gravidade de afecções nos cascos, como pododermatites, podendo até mesmo evoluir para uma hiperalgesia e, em casos mais intensos de claudicação e graves lesões nos membros, os animais podem inclusive assumir uma posição ajoelhada (Kaler et al., 2010).

Vacas com mastite constantemente apresentam dor intensa que pode gerar hiperalgesia, promovendo desconforto, aumento da temperatura do úbere, costumam dar chutes quando manejadas ou quando a região do úbere é palpada. Perda de peso, redução no consumo de alimentos, menos tempo de pastagem e redução na produção do leite também são observados nos animais acometidos (Roches et al., 2017). Já a ocorrência de metrite foi associada a presença de arqueamento do dorso em vacas submetidas a palpação retal e uterina, evidenciando a dor visceral gerada pela inflamação (Stojkov et al., 2015).

A vocalização em bovinos submetidos a procedimentos dolorosos é relatada, como marcação com ferro quente e castração/descorna, podendo ser observado vocalização em até $50 \%$ e $14 \%$ dos animais submetidos a estes procedimentos, respectivamente. É importante lembrar que o padrão de vocalização, bem como a intensidade podem variar entre as diferentes raças (Currah et al., 2009; Millman, 2013).

Levando em consideração a variedade de comportamentos expressos pelos bovinos ao sentirem dor, é importante salientarmos a dificuldade no reconhecimento e avaliação dessas alterações comportamentais, entretanto, a necessidade de conhecermos os comportamentos relacionados a sua presença na espécie é de fundamental importância para o direcionamento de estratégias que visem a redução da dor e a garantia de bem-estar para os animais (Oliveira et al., 2014; Remnant et al., 2017).

\section{Avaliação da dor}

A necessidade de se avaliar a dor constantemente deve-se à urgência em reconhecê-la e tratá-la o mais breve possível para minimizar o sofrimento dos animais. Com o intuito de minimizar os erros e a subjetividade das avaliações, as escalas de avaliação de dor constituem-se como importantes ferramentas para tal avaliação (Evangelista et al., 2019). A escala analógica visual e a escala numérica utilizadas para avaliar a dor em humanos e em outras espécies animais pode ser empregada em ruminantes. Entretanto estas escalas não possuem validação e são consideradas incompletas para a avaliação da dor (Channon et al., 2009). O conhecimento natural de cada espécie e as alterações comportamentais desencadeadas pela dor, são elementos importantes para a validação de uma escala. Nesse sentido, o etograma torna-se um instrumento de grande valor para descrever os comportamentos relacionados à dor. Nesta ferramenta, frequência e a duração de ocorrência dos comportamentos antes e após o estímulo doloroso normal e aqueles relacionados à dor são quantificados (Millman, 2013). Dentre as principais atividades e comportamentos naturais expressados por bovinos, citamos o ato de pastar como o mais comum, seguido de ruminar e descansar, que ocupam mais de $90 \%$ do dia de um animal. Os animais pastam predominantemente durante o dia e ruminam deitados mais frequentemente à noite (Kilgour et al., 2012).

Existem algumas escalas que foram desenvolvidas especialmente para avaliar a claudicação em bovinos, como a Escala numérica descritiva de claudicação para bovinos de Channon et al. (2009). Apesar de serem aplicadas na rotina clínica e experimental, esta escala apresenta uma determinada limitação quanto sua validade para detectar a dor e lesões nos membros de bovinos. Entretanto, a Escala numérica descritiva para avaliação de locomoção em bovinos, apresenta uma validade mais robusta, servindo também como um guia inicial para se aprofundar o diagnóstico e identificar a dor nos bovinos (Thomsen et al., 2008). Desde a elaboração das escalas numéricas descritivas para a avaliação da dor em bovinos, novas escalas foram criadas, baseadas em estudos comportamentais e com maior 
detalhamento quanto aos parâmetros avaliados, a exemplo da Escala Unidimensional da UnespBotucatu para avaliar a dor aguda pós-operatória em bovinos (Quadro 1) (Oliveira et al., 2014). Esta escala fora elaborada através da avaliação de filmagens realizadas antes e durante 24 horas de bovinos submetidos a orquiectomia. Os momentos de aferição foram, o basal, entre 1 e 4 horas após a cirurgia, uma hora após a intervenção analgésica e 24 horas após o procedimento. Com os resultados do estudo e análises, foi estabelecida uma escala confiável, válida e responsiva, que apresenta ponto de corte, ou escore, necessário para o resgate analgésico e que diferencia animais com ou sem dor (Oliveira et al., 2014).

Quadro 1. Escala Unidimensional da Unesp-Botucatu para avaliação da dor aguda pós-operatória em bovinos (Oliveira et al., 2014).

\begin{tabular}{|ll|}
\hline Item & Escore/Critério \\
\hline Locomoção & (0) move-se livremente, sem alterações de locomoção. \\
& (1) Move-se com restrição, e ao andar a linha dorsal pode estar normal ou arqueada e os \\
passos podem estar mais curtos. & (2) Reluta em se levantar ou, quando se levanta, o faz com dificuldade ou não se locomove. \\
Interação com o ambiente & (0) Ativo: atento aos estímulos ambientais táteis e/ou visuais e/ou sonoros; quando próximo \\
& aos outros animais, pode interagir /ou acompanhar o grupo. \\
& (1) Apático: pode permanecer próximo aos outros animais, mas quando estimulado interage \\
& pouco. \\
& (2) Apático: pode estar isolado ou não acompanhar os outros animais, não reage aos \\
& estímulos ambientais táteis e/ou visuais e/ou sonoros. \\
(0) Movimenta-se normalmente. & (1) Inquieto, movimenta-se acima do normal; ou deita e levanta com frequência. \\
Atividade & (2) Movimenta-se com menor frequência pelo piquete ou somente ao ser estimulado. \\
(0) Normorexia e/ou ruminação presente. \\
(1) Hiporexia. \\
(2) Anorexia. \\
Movimenta a cauda brusca e repentinamente. \\
Estende o pescoço e o corpo para frente, quando em decúbito ventral. \\
Movimenta e arqueia o dorso, quando em posição quadrupedal. \\
Escoiçeia e bate com o membro pélvico no chão. \\
Quando em decúbito ventral, ou ventrolateral ou lateral, estende um ou mais membros. \\
Quando em decúbito, a cabeça permanece próxima ou apoiada no solo. \\
Estende os membros pélvicos caudalmente. \\
Cabeça abaixo da linha da coluna. \\
Lambe a ferida cirúrgica. \\
(0) Todos os comportamentos acima relacionados estão ausentes. \\
(1) Presença de 1 dos comportamentos relacionados. \\
(2) Presença de 2 dos comportamentos relacionados.
\end{tabular}

Outra ferramenta importante para avaliar a dor em bovinos são as escalas de expressão facial, já desenvolvidas e relatadas em outras espécies como ovinos (McLennan et al., 2016), equinos (Dalla Costa et al., 2014) e gatos (Evangelista et al., 2019). Seu grande objetivo é identificar animais com e sem dor pela expressão facial. Nos animais com dor é possível notar alterações na posição das orelhas, que ficam mais tensas e apontadas para trás ou para baixo, enquanto os olhos ficam mais fechados ou o olhar fica ausente. Pode haver uma tensão da musculatura supraorbitária com formação de pregas na pele, a musculatura facial próxima à boca também fica contraída, a narina fica mais dilatada e os lábios contraídos (Gleerup et al., 2015).

Em um estudo realizados por Müller et al. (2019), com o objetivo de identificar alterações nas unidades de ação facial específicas em bovinos de corte da raça Nelore, pela análise de imagens fotográficas em 35 bovinos antes e durante a marcação com ferro quente, mostrou que a abertura da boca, orelhas voltadas para trás e narinas dilatadas são consideradas manifestações faciais associadas a dor nesses animais.

Apesar de existirem métodos de avaliação da presença de dor em bovinos, mais informações e estudos são necessários para elucidar o uso das escalas de expressão facial em bovinos com o intuito de reconhecer a dor e assim minimizar as dificuldades quanto a sua avaliação, diagnóstico e tratamento, permitindo intervenções rápidas e eficaz para amenizar o sofrimento dos animais. 


\section{Considerações finais}

O reconhecimento, avaliação e consequente tratamento da dor em bovinos é extremamente importante dentro dos sistemas de produção visando a supressão de situações de desconforto para os animais, bem-estar e melhorias na produção, entretanto são necessários mais estudos a respeito dos principais comportamentos e alterações expressas pelos bovinos quando apresentam dor e a aplicabilidade de ferramentas que venham auxiliar a avaliação. A rusticidade dos animais e seu grande porte não lhes exime a capacidade de sofrer estímulos dolorosos e a prática de procedimentos de manejo como castrações e descornas com a utilização de anestésicos locais ou analgésicos devem ser mais e mais incentivadas. É crescente a preocupação da sociedade com o tratamento moral e ético dos animais, sobretudo a respeito do bem-estar animal, que deve ser garantido.

\section{Referências}

Alvarez, L., Luna, J. B., Gamboa, D., Reyes, M., Sánchez, A., Terrazas, A., Rojas, S., \& Galindo, F. (2015). Cortisol and pain-related behavior in disbudded goat kids with and without cornual nerve block. Physiology \& Behavior, 138, 58-61. https://doi.org/10.1016/j.physbeh.2014.10.026.

Amaral, J. B., Pires, R. M. L., Ambrósio, L. A., \& Trevisan, G. (2017). Expressão facial, vocalização e posturas anômalas em bovinos submetidos à eletroejaculação convencional. PUBVET, 11, 10741187.

Antunes, M. I. P. P., Moreno, K., \& Grumadas, C. E. S. (2008). Avaliação e manejo da dor em cães e gatos com câncer-revisão. Arquivos de Ciências Veterinárias e Zoologia Da UNIPAR, 11(2), 113119.

Anzuino, K., Bell, N. J., Bazeley, K. J., \& Nicol, C. J. (2010). Assessment of welfare on 24 commercial UK dairy goat farms based on direct observations. Veterinary Record, 167(20), 774-780. https://doi.org/10.1136/vr.c5892.

Ballou, M. A., Sutherland, M. A., Brooks, T. A., Hulbert, L. E., Davis, B. L., \& Cobb, C. J. (2013). Administration of anesthetic and analgesic prevent the suppression of many leukocyte responses following surgical castration and physical dehorning. Veterinary Immunology and Immunopathology, 151(3-4), 285-293. https://doi.org/10.1016/j.vetimm.2012.11.018.

Battini, M., Vieira, A., Barbieri, S., Ajuda, I., Stilwell, G., \& Mattiello, S. (2014). Invited review: Animal-based indicators for on-farm welfare assessment for dairy goats. Journal of Dairy Science, 97(11), 6625-6648. https://doi.org/10.3168/jds.2013-7493.

Bergamasco, L., Coetzee, J. F., Mosher, R. M., \& Kotschwar, J. L. (2009). Quantitative electroencephalographic findings associated with nociception following surgical castration in conscious calves. American Association of Bovine Practitioners Proceedings of the Annual Conference, 197.

Bruijnis, M. R. N., Beerda, B., Hogeveen, H., \& Stassen, E. N. (2012). Assessing the welfare impact of foot disorders in dairy cattle by a modeling approach. Animal, 6(6), 962-970. https://doi.org/10.1017/S1751731111002606.

Cartopassi, S. R. G., \& Junior, E. M. (2012). Anestésicos locais. In D. T. Fantoni (Ed.), Tratamento da dor na clínica de pequenos animais. Elsevier.

Channon, A. J., Walker, A. M., Pfau, T., Sheldon, I. M., \& Wilson, A. M. (2009). Variability of Manson and Leaver locomotion scores assigned to dairy cows by different observers. Veterinary Record, 164(13), 388-392. https://doi.org/10.1136/vr.164.13.388.

Chapinal, N., de Passillé, A. M., Rushen, J., \& Wagner, S. (2010). Automated methods for detecting lameness and measuring analgesia in dairy cattle. Journal of Dairy Science, 93(5), 2007-2013. https://doi.org/http://dx.doi.org/10.3168/jds.2009-2803

Coetzee, J. F. (2013). Assessment and management of pain associated with castration in cattle. Veterinary Clinics: Food Animal Practice, 29(1), 75-101. https://doi.org/10.1016/j.cvfa.2012.11.012.

Currah, J. M., Hendrick, S. H., \& Stookey, J. M. (2009). The behavioral assessment and alleviation of pain associated with castration in beef calves treated with flunixin meglumine and caudal lidocaine 
epidural anesthesia with epinephrine. The Canadian Veterinary Journal, 50(4), 375-382.

Dalla Costa, E., Minero, M., Lebelt, D., Stucke, D., Canali, E., \& Leach, M. C. (2014). Development of the Horse Grimace Scale (HGS) as a pain assessment tool in horses undergoing routine castration. PLoS One, 9(3), e92281. https://doi.org/10.1371/journal.pone.0092281.

Davidson, E. N. B., Van Caam, A. P. M., Vitters, E. L., Bennink, M. B., Thijssen, E., Van Den Berg, W. B., Koenders, M. I., Van Lent, P., Van De Loo, F. A. J., \& Van Der Kraan, P. M. (2015). TGF- $\beta$ is a potent inducer of Nerve Growth Factor in articular cartilage via the ALK5-Smad2/3 pathway. Potential role in OA related pain? Osteoarthritis and Cartilage, 23(3), 478-486. https://doi.org/10.1016/j.joca.2014.12.005.

Evangelista, M. C., Watanabe, R., Leung, V. S. Y., Monteiro, B. P., O’Toole, E., Pang, D. S. J., \& Steagall, P. V. (2019). Facial expressions of pain in cats: the development and validation of a Feline Grimace Scale. Scientific Reports, 9(1), 1-11. https://doi.org/10.1038/s41598-019-55693-8.

Fajt, V. R., Wagner, S. A., \& Norby, B. (2011). Analgesic drug administration and attitudes about analgesia in cattle among bovine practitioners in the United States. Journal of the American Veterinary Medical Association, 238(6), 755-767. https://doi.org/10.2460/javma.238.6.755.

Flecknell, P. A. (2008). Analgesia from a veterinary perspective. British Journal of Anaesthesia, 101(1), 121-124. https://doi.org/https://doi.org/10.1093/bja/aen087.

Fulwider, W. K., Grandin, T., Rollin, B. E., Engle, T. E., Dalsted, N. L., \& Lamm, W. D. (2008). Survey of dairy management practices on one hundred thirteen north central and northeastern United States dairies. Journal of Dairy Science, 91(4), 1686-1692. https://doi.org/10.3168/jds.2007-0631.

Futro, A., Masłowska, K., \& Dwyer, C. M. (2015). Ewes direct most maternal attention towards lambs that show the greatest pain-related behavioural responses. PLoS One, 10(7), e0134024. https://doi.org/10.1371/journal.pone.0134024.

Galatos, A. D. (2011). Anesthesia and Analgesia in Sheep and Goats. Veterinary Clinics of North America: $\quad$ Food Animal Practice, 27(1), 47-59. https://doi.org/http://dx.doi.org/10.1016/j.cvfa.2010.10.007

Giuliodori, M. J., Magnasco, R. P., Becu-Villalobos, D., Lacau-Mengido, I. M., Risco, C. A., \& de la Sota, R. L. (2013). Metritis in dairy cows: Risk factors and reproductive performance. Journal of Dairy Science, 96(6), 3621-3631. https://doi.org/10.3168/jds.2012-5922.

Gleerup, K. B., Andersen, P. H., Munksgaard, L., \& Forkman, B. (2015). Pain evaluation in dairy cattle. Applied Animal Behaviour Science, 171, 25-32.

González, L. A., Schwartzkopf-Genswein, K. S., Caulkett, N. A., Janzen, E., McAllister, T. A., Fierheller, E., Schaefer, A. L., Haley, D. B., Stookey, J. M., \& Hendrick, S. (2010). Pain mitigation after band castration of beef calves and its effects on performance, behavior, Escherichia coli, and salivary cortisol. Journal of Animal Science, 88(2), 802-810.

Grimm, K., Lamont, L., Tranquilli, W., Greene, S., \& Robertson, S. (2015). Anestesiologia e analgesia em veterinária. Editora Roca.

Guesgen, M. J., Beausoleil, N. J., Minot, E. O., Stewart, M., Jones, G., \& Stafford, K. J. (2011). The effects of age and sex on pain sensitivity in young lambs. Applied Animal Behaviour Science, 135(12), 51-56. https://doi.org/http://dx.doi.org/10.1016/j.applanim.2011.09.008

Jimenez, R. E., Adcock, S. J. J., \& Tucker, C. B. (2019). Acute pain responses in dairy calves undergoing cornual nerve blocks with or without topical anesthetic. Journal of Dairy Science, 102(4), 34313438. https://doi.org/10.3168/jds.2018-15445.

Kaler, J., Medley, G. F., Grogono-Thomas, R., Wellington, E. M. H., Calvo-Bado, L. A., Wassink, G. J., King, E. M., Moore, L. J., Russell, C., \& Green, L. E. (2010). Factors associated with changes of state of foot conformation and lameness in a flock of sheep. Preventive Veterinary Medicine, 97(34), 237-244.

Keating, S. C. J., Thomas, A. A., Flecknell, P. A., \& Leach, M. C. (2012). Evaluation of EMLA cream for preventing pain during tattooing of rabbits: changes in physiological, behavioural and facial expression responses. PloS One, 7(9), e44437. https://doi.org/10.1371/journal.pone.0044437.

Kilgour, R. J., Uetake, K., Ishiwata, T., \& Melville, G. J. (2012). The behaviour of beef cattle at pasture. 
$\begin{array}{llll}\text { Applied Animal } & \text { Behaviour } & \text { Science, } & \text { 138(1-2), }\end{array}$ https://doi.org/http://dx.doi.org/10.1016/j.applanim.2011.12.001

Kotschwar, J. L., Coetzee, J. F., Anderson, D. E., Gehring, R., KuKanich, B., \& Apley, M. D. (2009). Analgesic efficacy of sodium salicylate in an amphotericin B-induced bovine synovitis-arthritis model. Journal of Dairy Science, 92(8), 3731-3743. https://doi.org/10.3168/jds.2009-2058.

Langford, D. J., Bailey, A. L., Chanda, M. L., Clarke, S. E., Drummond, T. E., Echols, S., Glick, S., Ingrao, J., Klassen-Ross, T., \& LaCroix-Fralish, M. L. (2010). Coding of facial expressions of pain in the laboratory mouse. Nature Methods, 7(6), 447-449.

Laven, R., Chambers, P., \& Stafford, K. (2012). Using non-steroidal anti-inflammatory drugs around calving: Maximizing comfort, productivity and fertility. The Veterinary Journal, 192(1), 8-12. https://doi.org/10.1016/j.tvj1.2011.10.023.

Leslie, K. E., \& Petersson-Wolfe, C. S. (2012). Assessment and management of pain in dairy cows with clinical mastitis. Veterinary Clinics: Food Animal Practice, 28(2), 289-305. https://doi.org/10.1016/j.cvfa.2012.04.002.

Lorena, S. E. R. S., Luna, S. P. L., Lascelles, B. D. X., \& Corrente, J. E. (2013). Attitude of Brazilian veterinarians in the recognition and treatment of pain in horses and cattle. Veterinary Anaesthesia and Analgesia, 40(4), 410-418. https://doi.org/10.1111/vaa.12025.

Mathews, K., Kronen, P. W., Lascelles, D., Nolan, A., Robertson, S., Steagall, P. V. M., Wright, B., \& Yamashita, K. (2015). Guidelines for recognition, assessment and treatment of pain. The Veterinary Nurse, 6(3), 164-173.

McLennan, K. M, Rebelo, C. J. B., Corke, M. J., Holmes, M. A., Leach, M. C., \& Constantino-Casas, F. (2016). Development of a facial expression scale using footrot and mastitis as models of pain in sheep. Applied Animal Behaviour Science, 176, 19-26. https://doi.org/10.1016/j.applanim.2016.03.005.

McLennan, K. M. (2018). Why pain is still a welfare issue for farm animals, and how facial expression could be the answer. Agriculture, 8(8), 127. https://doi.org/10.3390/agriculture8080127.

Millman, S. T. (2013). Behavioral responses of cattle to pain and implications for diagnosis, management, and animal welfare. Veterinary Clinics: Food Animal Practice, 29(1), 47-58. https://doi.org/10.1016/j.cvfa.2012.11.007.

Moggy, M. A., Pajor, E. A., Thurston, W. E., Parker, S., Greter, A. M., Schwartzkopf-Genswein, K. S., Campbell, J. R., \& Windeyer, M. C. (2017). Management practices associated with pain in cattle on western Canadian cow-calf operations: A mixed methods study. Journal of Animal Science, 95(2), 958-969. https://doi.org/10.2527/jas2016.0949.

Mosher, R. A., Wang, C., Allen, P. S., \& Coetzee, J. F. (2013). Comparative effects of castration and dehorning in series or concurrent castration and dehorning procedures on stress responses and production in Holstein calves. Journal of Animal Science, 91(9), 4133-4145. https://doi.org/10.2527/jas.2012-6007.

Müller, B. R., Soriano, V. S., Bellio, J. C. B., \& Molento, C. F. M. (2019). Facial expression of pain in Nellore and crossbred beef cattle. Journal of Veterinary Behavior, 34, 60-65. https://doi.org/10.1016/j.jveb.2019.07.007.

Newby, N. C., Pearl, D. L., LeBlanc, S. J., Leslie, K. E., von Keyserlingk, M. A. G., \& Duffield, T. F. (2013). Effects of meloxicam on milk production, behavior, and feed intake in dairy cows following assisted calving. Journal of Dairy Science, 96(6), 3682-3688. https://doi.org/http://dx.doi.org/10.3168/jds.2012-6214

Oliveira, F. A., Luna, S. P. L., Amaral, J. B., Rodrigues, K. A., Sant'Anna, A. C., Daolio, M., \& Brondani, J. T. (2014). Validation of the UNESP-Botucatu unidimensional composite pain scale for assessing postoperative pain in cattle. BMC Veterinary Research, 10(1), 1-14. https://doi.org/10.1016/j.vaa.2016.11.008.

Opsomer, G. (2015). Metritis and endometritis in high yielding dairy cows. Revista Brasileira de Reprodução Animal, 39(1), 164-172.

Pauly, C., White, B. J., Coetzee, J. F., Robert, B., Baldridge, S., \& Renter, D. G. (2012). Evaluation of 
analgesic protocol effect on calf behavior after concurrent castration and dehorning. International Journal of Applied Research in Veterinary Medicine, 10(1), 54-61.

Randall, L. V, Green, M. J., Chagunda, M. G. G., Mason, C., Green, L. E., \& Huxley, J. N. (2016). Lameness in dairy heifers; impacts of hoof lesions present around first calving on future lameness, milk yield and culling risk. Preventive Veterinary Medicine, 133, 52-63. https://doi.org/10.1016/j.prevetmed.2016.09.006.

Remnant, J. G., Tremlett, A., Huxley, J. N., \& Hudson, C. D. (2017). Clinician attitudes to pain and use of analgesia in cattle: where are we 10 years on? Veterinary Record, 181(15), 400. https://doi.org/10.1136/vr.104428.

Roches, A. B., Faure, M., Lussert, A., Herry, V., Rainard, P., Durand, D., \& Foucras, G. (2017). Behavioral and patho-physiological response as possible signs of pain in dairy cows during Escherichia coli mastitis: A pilot study. Journal of Dairy Science, 100(10), 8385-8397. https://doi.org/10.3168/jds.2017-12796.

Shearer, J. K., Stock, M. L., Van Amstel, S. R., \& Coetzee, J. F. (2013). Assessment and management of pain associated with lameness in cattle. Veterinary Clinics: Food Animal Practice, 29(1), 135156. https://doi.org/10.1016/j.cvfa.2017.02.009.

Stewart, M., Verkerk, G. A., Stafford, K. J., Schaefer, A. L., \& Webster, J. R. (2010). Noninvasive assessment of autonomic activity for evaluation of pain in calves, using surgical castration as a model. Journal of Dairy Science, 93(8), 3602-3609. https://doi.org/10.3168/jds.2010-3114.

Stilwell, G., Schubert, H., \& Broom, D. M. (2014). Effects of analgesic use postcalving on cow welfare and production. Journal of Dairy Science, 97(2), 888-891. https://doi.org/10.3168/jds.2013-7100.

Stock, M. L., Baldridge, S. L., Griffin, D., \& Coetzee, J. F. (2013). Bovine dehorning: Assessing pain and providing analgesic management. Veterinary Clinics: Food Animal Practice, 29(1), 103-133. https://doi.org/10.1016/j.cvfa.2012.11.001.

Stojkov, J., Von Keyserlingk, M. A. G., Marchant-Forde, J. N., \& Weary, D. M. (2015). Assessment of visceral pain associated with metritis in dairy cows. Journal of Dairy Science, 98(8), 5352-5361. https://doi.org/10.3168/jds.2014-9296.

Sutherland, M. A., Ballou, M. A., Davis, B. L., \& Brooks, T. A. (2013). Effect of castration and dehorning singularly or combined on the behavior and physiology of Holstein calves. Journal of Animal Science, 91(2), 935-942. https://doi.org/10.2527/jas.2012-5190.

Theurer, M. E., Amrine, D. E., \& White, B. J. (2013). Remote Noninvasive Assessment of Pain and Health Status in Cattle. Veterinary Clinics of North America: Food Animal Practice, 29(1), 59-74. https://doi.org/http://dx.doi.org/10.1016/j.cvfa.2012.11.011

Thomsen, P. T., Munksgaard, L., \& Tøgersen, F. A. (2008). Evaluation of a lameness scoring system for dairy cows. Journal of Dairy Science, 91(1), 119-126. https://doi.org/0.3168/jds.2007-0496.

White, B. J., Coetzee, J. F., Renter, D. G., Babcock, A. H., Thomson, D. U., \& Andresen, D. (2008). Evaluation of two-dimensional accelerometers to monitor behavior of beef calves after castration. American Journal of Veterinary Research, 69(8), 1005-1012. https://doi.org/10.2460/ajvr.69.8.1005.

Wittrock, J. M., Proudfoot, K. L., Weary, D. M., \& Von Keyserlingk, M. A. G. (2011). Metritis affects milk production and cull rate of Holstein multiparous and primiparous dairy cows differently. Journal of Dairy Science, 94(5), 2408-2412. https://doi.org/10.3168/jds.2010-3697.

Histórico do artigo:

Recebido: 2 de dezembro de 2020 . Aprovado: 14 de janeiro de 2021. Disponível online: 16 de março de 2021.
Licenciamento: Este artigo é publicado na modalidade Acesso Aberto sob a licença Creative Commons Atribuição 4.0 (CC-BY 4.0), a qual permite uso irrestrito, distribuição, reprodução em qualquer meio, desde que o autor e a fonte sejam devidamente creditados. 